LAWRENCE LIVERMORE N A T IO N A L LABORATORY

\section{Properties of Fluid Deuterium \\ under Double-Shock Compression to Several Mbar}

T. R. Boehly, D. G. Hicks, P. M. Celliers, T. J. B.

Collins, J. H. Eggert, S. J. Moon, E. Vianello, D. D. Meyerhofer, G. W. Collins

March 1, 2004

Physics of Plasmas 
This document was prepared as an account of work sponsored by an agency of the United States Government. Neither the United States Government nor the University of California nor any of their employees, makes any warranty, express or implied, or assumes any legal liability or responsibility for the accuracy, completeness, or usefulness of any information, apparatus, product, or process disclosed, or represents that its use would not infringe privately owned rights. Reference herein to any specific commercial product, process, or service by trade name, trademark, manufacturer, or otherwise, does not necessarily constitute or imply its endorsement, recommendation, or favoring by the United States Government or the University of California. The views and opinions of authors expressed herein do not necessarily state or reflect those of the United States Government or the University of California, and shall not be used for advertising or product endorsement purposes. 


\title{
Properties of Fluid Deuterium under Double-Shock Compression to Several Mbar
}

\author{
T. R. Boehly, ${ }^{1}$ D. G. Hicks, ${ }^{2}, *$ P. M. Celliers, ${ }^{2}$ T. J. B. Collins, ${ }^{1}$ J. H. \\ Eggert, ${ }^{2}$ S. J. Moon, ${ }^{2}$ E. Vianello, ${ }^{1}{ }^{\dagger}$ D. D. Meyerhofer, ${ }^{1,}{ }^{\ddagger}$ and G. W. Collins ${ }^{2}$ \\ ${ }^{1}$ Laboratory for Laser Energetics, University of Rochester, NY 14623 \\ ${ }^{2}$ Lawrence Livermore National Laboratory, Livermore, CA 94550
}

(Dated: February 3, 2004)

\begin{abstract}
The compressibility of fluid deuterium up to several Mbar has been probed using laser-driven shock waves reflected from a quartz anvil. Combining high-precision $(\sim 1 \%)$ shock velocity measurements with the double-shock technique, where differences in equation of state (EOS) models are magnified, has allowed us to accurately discriminate between various theoretical predictions. Our data are consistent with EOS models that show approximately fourfold compression on the principal Hugoniot from 0.7 to 1 Mbar; however, our results indicate that deuterium has a higher compressibility than predicted by these models for single shock pressures between 1 and 2.5 Mbar.
\end{abstract}

PACS numbers: $62.50 .+\mathrm{p}, 64.30 .+\mathrm{t}$

The equation of state of hydrogen at pressures of a few Megabar, temperatures of a few eV, and compressions of up to several times liquid density has been a source of on-going experimental [1-3] and theoretical [5-11] controversy. Understanding the properties of hydrogen under such conditions is fundamental to the modelling of massive planets, brown dwarfs, and inertial confinement fusion. At present, access to these dense, high-pressure states can only be achieved using shock waves; Hugoniot measurements are thus the primary tool for constraining dense hydrogen equation of state (EOS) models.

Dense, shocked deuterium is expected to reach a limiting compression of 4 at several Mbars when its state has become compressed and heated to that of a monatomic ideal gas [5-7]. Before reaching this limit, higher compressions can be achieved if deuterium remains mostly molecular [7], with some models predicting an associated plasma phase transition (PPT) to a higher density state [6]. Such non-ideal behavior (as evidenced by greater than 4-fold compression) was predicted to begin between 0.5 and 1 Mbar $[6,7]$. The first experiments to study deuterium above $0.2 \mathrm{Mbar}$ used laser-driven shock waves and a new, absolute measurement technique; results showed 5 to 6 -fold compression between 0.5 and 2.5 Mbar [1] suggesting that deuterium remains molecular at these high pressures [7]. Further laser-driven shock experiments by Mostovych et al. [2], using a doubleshock approach up to first shock pressures of $1.3 \mathrm{Mbar}$, also found behavior consistent with 5 to 6 -fold compression. However, subsequent studies using magneticallydriven flyer plates and a relative measurement of particle speed based on aluminum impedance-matching, found only slightly over 4-fold compression between 0.4 and 1 Mbar [3], in accordance with a new SESAME model developed by Kerley [8] (which, for clarity, we refer to as the Kerley98 model) and $a b$ initio models $[10,11]$. The achievement of only 4-fold compression in these latest experiments was interpreted as an indication that deuterium had reached an ideal, monatomic state below 1
Mbar [12], casting doubt on the validity of the original laser-driven shock experiments.

To address this controversy, we have undertaken a series of highly precise, laser-driven shock experiments to study the double-shock behavior of fluid deuterium at pressures between 0.7 and 2.5 Mbar. We cover a wider range of pressures and have significantly higher measurement precision than the original double-shock experiments by Mostovych et al. [2], using an approach that determines shock velocities to $1 \%$ uncertainty and essentially eliminates any systematic errors due to shock unsteadiness and non-planarity. The advantage of the double-shock approach is that it magnifies the expected differences in the experimental observables (i.e. measured shock speeds) predicted by various EOS models, thus providing a more sensitive platform for discriminating between models. Up to pressures of 1 Mbar, our results are consistent with models predicting only $\sim 4$ fold single-shock compression $[8,10]$ - in agreement with the magnetically-driven flyer plate experiments [3] but in disagreement with both the earlier laser-driven shock experiments [1, 2]. However, above 1 Mbar, beyond pressures explored by the flyer plate studies, we find that deuterium undergoes further compression. This confirms the trend exhibited by the earlier laser-driven shock experiments of showing greater than 4-fold compression above $1 \mathrm{Mbar}$, although our high-precision results indicate that the enhanced compressions are less than 6-fold and are restricted to a narrower pressure range (1 - $2.5 \mathrm{Mbar})$ than shown in those earlier studies. Such increased compressibility is strong evidence that deuterium at $1 \mathrm{Mbar}$ has not yet reached its ideal, monatomic gas limit.

This experiment was performed on the OMEGA laser at the University of Rochester, a neodymium-doped phosphate glass system that operates with frequencytripled, $0.35 \mu \mathrm{m}$ light [13]. To generate the shock pressures explored in these experiments, laser energies of 440 to $3100 \mathrm{~J}$ were delivered using a square pulse $3.7 \mathrm{~ns}$ in duration. The laser focal region was smoothed using dis- 


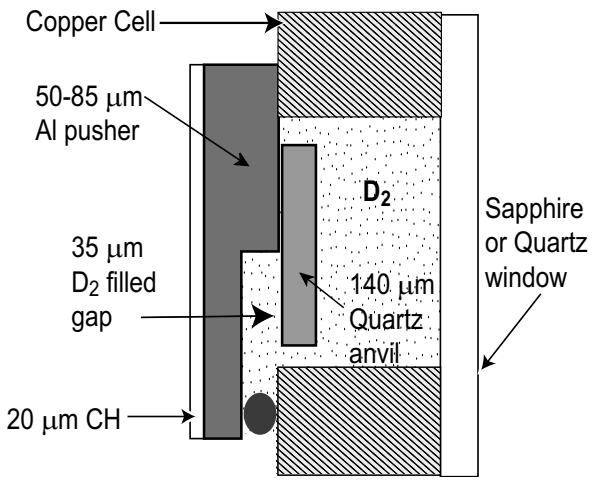

FIG. 1: Characteristic cryogenic deuterium target design. Dimensions are for one of the three types of target.

tributed phase plates, producing a uniformly-irradiated spot $800 \mu \mathrm{m}$ in diameter. Targets consisted of a z-cut, $\alpha$-quartz anvil mounted on the upper step of a diamondturned aluminum pusher which was attached to a copper cell filled with cryogenic deuterium (see Fig. 1). A plastic ablator was used to reduce hard x-ray generation. Three different thicknesses were used for the ablatorpusher combination: $20 \mu \mathrm{m}$ of $\mathrm{CH}$ on a $90-130 \mathrm{Al}$ step (90 $\mu \mathrm{m}$ lower step and $130 \mu \mathrm{m}$ upper step); $20 \mu \mathrm{m}$ of $\mathrm{CH}$ on a $50-85 \mu \mathrm{m} \mathrm{Al} \mathrm{step,} \mathrm{and} 20 \mu \mathrm{m} \mathrm{CH}$ plus $80 \mu \mathrm{m}$ of $\mathrm{CH}-\mathrm{Br}$ (plastic with $2 \% \mathrm{Br}$ by atomic weight) on a $50-85 \mu \mathrm{m} \mathrm{Al}$ step. The quartz anvil was glued to the upper step with a glue thickness of $\sim 1 \mu \mathrm{m}$ and hung over the lower step as shown in Fig. 1. The deuterium sample explored in this experiment is trapped within the $35-40 \mu \mathrm{m}$ region between the quartz anvil and the thin $\mathrm{Al}$ plate. By observing the solid-liquid transition in deuterium and using the well-known properties of deuterium on the saturation line [16], we determined that the deuterium density was $0.174 \mathrm{~g} / \mathrm{cm}^{3}$. At this density and at the probe laser wavelength of $532 \mathrm{~nm}$, the index of refraction was calculated to be 1.1381 [16]. At room temperature, the density of quartz was measured to be $2.65 \mathrm{~g} / \mathrm{cm}^{3}$ and the refractive index along its c-axis at $532 \mathrm{~nm}$ was found to be 1.547 . Because quartz has such a low thermal expansivity, its density changes by only $0.5 \%$ and its refractive index by $0.1 \%$ when cooled to $<20 \mathrm{~K}$, changes that are negligible for our purposes and can be neglected.

The shock diagnostic was a line-imaging Velocity Interferometer System for Any Reflector (VISAR) [14, 15] which measures the Doppler shift of a moving reflector. Two VISAR's with different velocity sensitivities were used to resolve $2 \pi$ phase shift ambiguities that occur at shock break-out from the aluminum and upon transit of the shock front from deuterium into quartz. The velocity sensitivities for the two VISAR instruments were 6.069 and $14.138 \mu \mathrm{m} / \mathrm{ns} /$ fringe for deuterium and 4.465 and $10.400 \mu \mathrm{m} / \mathrm{ns} /$ fringe for quartz. Post-processing of the VISAR images can determine the fringe position to $\sim 5$ $\%$ of a fringe; since the measured shock velocities are 25
- $45 \mu \mathrm{m} / \mathrm{ns}$ in deuterium and $14-24 \mu \mathrm{m} / \mathrm{ns}$ in quartz, multiple fringe shifts allow the precision of the shock velocity measurement to be $\sim 1 \%$. The probe source was an injection-seeded, Q-switched, yttrium-aluminum garnet laser, operating at a wavelength of $532 \mathrm{~nm}$ with a pulse length of $\sim 25 \mathrm{~ns}$. Streak cameras with temporal windows of $\sim 3$ ns were used to detect the reflected probe signal. The time resolution of the VISAR and streak camera system was about 40-50 ps.

A sample VISAR trace is shown in Fig. 2(a) and the resulting velocity profile inferred from the fringe positions is given in Fig. 2(b). The three clear events observed in these records are marked by fringe (and hence velocity) shifts: The first shift represents the velocity jump that occurs when the shock crosses the aluminum-deuterium interface; the second shift, at time $t_{x}$, corresponds to the drop in shock velocity as the shock moves across the deuterium-quartz interface. Shock velocities immediately before and after the shock crosses the $\mathrm{D}_{2}$-quartz interface are the primary observables used in this work. The third shift is the jump in velocity observed in quartz when the first shock reverberating in the compressed deuterium gap catches the leading shock front in quartz.

To extract the velocity profile we average the phase information at each time over a $20-30 \mu \mathrm{m}$ region. To determine shock velocities at the deuterium-quartz interface, we take linear fits a few hundred ps before and after $t_{x}$ and extrapolate them to $t_{x}$. This eliminates ambiguities due to slight blurring of the measured velocity in a \pm 25 ps time window centered on $t_{x}$ caused by the resolution of the VISAR and streak camera system. Fig. 3 plots the results in terms of the primary experimental observables: the shock speed in deuterium and quartz.

In order to compare these observations with EOS models it is necessary to know the high-pressure $U_{s}-U_{p}$ relation for quartz. To determine this we performed extensive laser-driven shock measurements on quartz [17], complementing earlier data reported by Russian workers obtained using nuclear explosives [18], and found that $U_{s}=3.915+1.297 U_{p}$. Taking into account the errors in the fit coefficients this is in excellent agreement with the relation found in the early Russian work $\left(U_{s}=4.200+1.280 U_{p}\right)[19]$ over the range of pressures in our study. Our measurements were performed using impedance-matching with an aluminum standard for which we utilized the experimentally derived aluminum Hugoniot relation given by $U_{s}(A l)=6.541+$ $1.158 U_{p}(A l)$ [19]; because quartz and aluminum are closely impedance matched the release was accurately calculated from the reflected Hugoniot [20]. The results change very little - and only then at the very highest pressures - if we instead utilize a SESAME EOS for aluminum [21].

Silica is well known to possess a number of polymorphic phase transitions in the solid regime below 1 Mbar [22]. Above $\sim 1$ Mbar, where silica is a fluid, quartz Hugoniot 

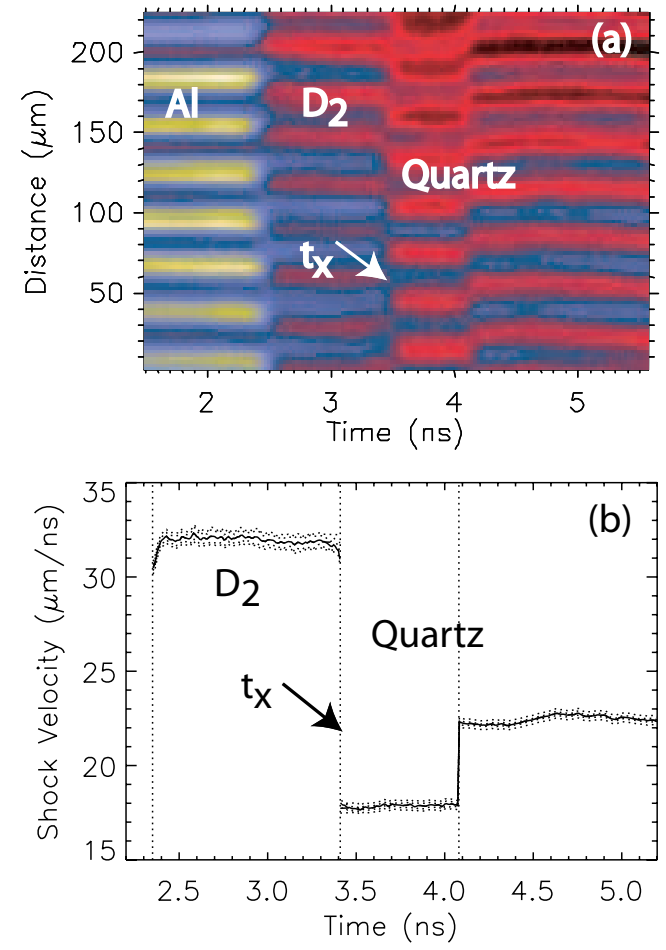

FIG. 2: (a) Sample VISAR trace showing the signal from the reflecting shock front in deuterium and quartz. (b) Resulting velocity profile extracted from the VISAR trace in (a). Dotted lines above and below the main trace indicate the error at each time step. The shock traverses the deuterium-quartz interface at time $t_{x}$.

measurements have not shown any indication of a subsequent structural change [18]. Our data begin at $\sim 2.5$ Mbar and closely follow a linear fit in $U_{s}-U_{p}$.

Using this fit and the impedance-matching conditions at the deuterium-quartz interface we calculate the reflected shock curves for the different EOS models. These are shown in Fig. 3 where the thickness of the lines represents the uncertainty in the linear fit to the quartz Hugoniot (for clarity, the PIMC results, which being close to the Kerley98 predictions, are shown as squares). Plotting the data in terms of the experimental observables thus allows uncertainties in the quartz Hugoniot (a systematic error in all the data) to be separated from measurement errors in the deuterium re-shock data (which are given by the error bars on the data points). Note that a clear trend is apparent from Fig. 3: softer EOS models (blue and green lines) [6, 7], which have roughly 6-fold single-shock compression, predict higher final reshock pressures than do stiffer models (red and black lines, black squares) $[5,8,10]$, which show only 4 - 4.5fold compression.

Results indicate that the compressibility of deuterium at single-shock pressures between 0.7 and 2.5 Mbar cannot be adequately described over this range by any of the models shown. Below 1.0 Mbar, the PIMC [10] and

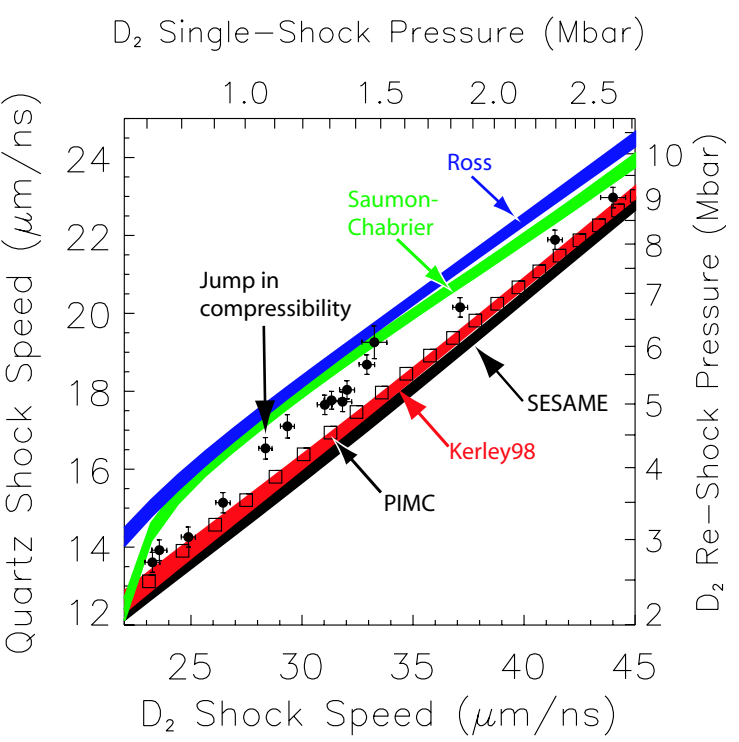

FIG. 3: Double-shock data from this study (dark circles with error bars) suggesting a jump in compressibility at around 1 Mbar. Predictions for 5 different EOS's are shown: SESAME [5], Kerley98 [8], Saumon-Chabrier [6], Ross [7], and PIMC [10] (black squares) where the line thicknesses correspond to the quartz Hugoniot uncertainty. The softer models (SaumonChabrier, Ross) which have 6-fold maximum compressibility on the principal Hugoniot, predict higher final re-shock pressures than the stiffer models (Kerley98, SESAME, PIMC), which have 4 - 4.5-fold compressibility; between 1 and 2.5 Mbar, our data lie in between. The estimated $\mathrm{D}_{2}$ single-shock and re-shock pressures on the top and right axes are based on the PIMC model for $\mathrm{D}_{2}$ and the measured quartz Hugoniot respectively.

Kerley98 [8] models, which predict 4 - 4.5-fold compression on the principal Hugoniot, are consistent with our data; around 1 Mbar, our data show a sudden jump in compressibility (see Fig. 3) and, up to $\sim 2.5$ Mbar, lie in between the predictions of softer $[6,7]$ and stiffer $[5,8,10]$ models, suggesting about 5 -fold compression in this intermediate regime; at 2.5 Mbar, the results show a return to stiffer behavior and are again consistent with the PIMC and Kerley98 calculations.

We have considered a number of potential systematic effects that could compromise our data and address each of them below. The steadiness of shock wave velocities in our experiments varied from shot to shot, depending on the quality of the laser drive, ranging from fractions of a percent to several percent over a few nanoseconds. Our new technique of determining shock velocities at essentially a single point in time using continuous measurements is not affected by such variations, unlike the transit time measurements which were used in the earlier re-shock experiments [2]. To establish this we performed extensive hydrodynamic simulations of our experimental arrangement using shock waves with a wide range of unsteadiness - rising and decaying. We saw no deviations 
from the steady shock case if the velocities were extrapolated to time $t_{x}$. This is confirmed experimentally where we observe no difference between the shots which were essentially steady and those which had several percent unsteadiness.

Shock non-planarity is also a potential problem, especially for an experiment that requires measurement of a break-out event at spatially-separated positions [2]. However, since our measurement is performed at a localized point in space, we are not subject to such errors. Non-planarity could affect our measurements if the wave is incident on the deuterium-quartz interface at a large enough angle to undergo significant refraction. Based on our measurement of the small curvature observed at the deuterium-quartz interface, we infer that the largest incident angles present in our experiments are 3 degrees to target normal. The resulting change in the projected shock speed is less than $0.1 \%$ and can be neglected.

$\mathrm{X}$-ray pre-heating of our target system is a process which would tend to make our data look less compressible. Using an etalon sensitive to motions as low as 0.1 $\mu \mathrm{m} / \mathrm{ns}$, we did not observe any expansion of the aluminum pusher prior to shock break-out. In addition, for targets shot at similar laser energies, we did not see any difference in the results whether we use a $50 \mu \mathrm{m}$ or 90 $\mu \mathrm{m}$ thick aluminum pusher. Since the attenuation length for a $1.55 \mathrm{keV}$ x-ray (just below the K-edge of aluminum) is $10 \mu \mathrm{m}$, the extra $40 \mu \mathrm{m}$ of $\mathrm{Al}$ would be expected to reduce the x-ray fluence by a factor of 50 . The absence of any difference between results from these two targets indicates that $\mathrm{x}$-ray pre-heat is negligible for these experiments.

Using a laser-driven, double-shock technique, we have performed the highest-precision experiments to date on deuterium shocked to initial pressures between 0.7 and 2.5 Mbar. For pressures up to 1 Mbar we find for the first time that laser-driven, magnetically-driven flyer plate [3], and high-explosive driven [4] shock experiments all provide consistent results. At higher pressures, between 1 and 2.5 Mbar, our results indicate that deuterium exhibits a jump in compressibility. This trend toward higher compressibility is similar to that measured by previous laser-driven shock experiments at these pressures although our more precise data indicate that the enhanced compression is less than 6-fold and does not begin until pressures are greater than 1 Mbar. This indicates that the simple view of deuterium having reached an ideal, monatomic state by 0.5 Mbar [12] is incorrect. We note that such an increase in compressibility above 1 Mbar was recently predicted by ab initio models [23]. Given the initial controversy surrounding the measurements below 1 Mbar, which we now believe has been resolved by using higher-precision laser-shock experiments, it is critical that these higher pressure results be reproduced on other experimental platforms.

We thank G. I. Kerley and B. Militzer for providing model calculations and the OMEGA target fabrication and operations staff for their efforts during these experiments. This work was performed under the auspices of the U.S. D.O.E. by LLNL under Contract No. W-7405ENG-48 and by the University of Rochester under Cooperative Agreement No. DE-FC03-92SF19460.

* Corresponding author: hicks13@llnl.gov

$\dagger$ Also Department of Physics and Astronomy.

$¥$ Also Department of Physics and Astronomy and Department of Mechanical Engineering.

[1] L. B. Da Silva et al., Phys. Rev. Lett. 78, 483 (1997); G. W. Collins et al., Science 281, 1178 (1998); G. W. Collins et al., Phys. Plasmas 5, 1864 (1998).

[2] A. N. Mostovych et al., Phys. Rev. Lett. 85, 3870 (2000); A. N. Mostovych et al., Phys. Plasmas 8, 2281 (2001).

[3] M. D. Knudson et al., Phys. Rev. Lett. 87, 225501 (2001); M. D. Knudson et al., Phys. Rev. Lett. 90, 035505 (2003).

[4] S. I. Belov et al., JETP Lett. 76, 433 (2002).

[5] G. I. Kerley, A theoretical equation of state for deuterium (Los Alamos Laboratory Rep. LA-4776, Los Alamos, NM, 1972); J. Chem. Phys. 73, 460 (1980).

[6] D. Saumon and G. Chabrier, Phys. Rev. A 46, 2084 (1992); D. Saumon, G. Chabrier, H. M. Van Horn, Astrophys. J., Suppl. Ser. (99), 713 (1995).

[7] M. Ross, Phys. Rev. B 58669 (1998)

[8] G. I. Kerley, private communication. This model has also been referred to as the SESAME model [3] but shows softer behavior than the original SESAME [5] referred to in earlier publications [1].

[9] D. A. Young, Shock Compression of Condensed Matter - 1999, M. D. Furnish, L. C. Chhabildas, R. S. Hixson eds., 53 (AIP, 1999).

[10] B. Militzer and D. M. Ceperley, Phys. Rev. Lett. 85, 1890 (2000); B. Militzer et al., Phys. Rev. Lett. 87, 275502 (2001).

[11] T. Lenosky et al., Phys. Rev. B 61, 1 (2000); L. Collins et al., Phys. Rev. B 63, 184110 (2001).

[12] W. J. Nellis, Phys. Rev. Lett. 89, 165502-1 (2002)

[13] T. R. Boehly et al., Opt. Comm. 133, 495 (1997).

[14] L. M. Barker and R. E. Hollenbach, J. Appl Phys. 43, 4669 (1972).

[15] P. M. Celliers et al., Appl. Phys. Lett. 73, 1320 (1998).

[16] P. C. Souers, Hydrogen Properties for Fusion Energy, Univ. of California Press, Berkeley (1986).

[17] D. G. Hicks et al., in preparation.

[18] L. V. Al'tshuler, R. F. Trunin, and G. V. Simakov, Acad. Sciences USSR, Izvestiya, Phys. Solid Earth, No. 1, 657 (1965); R. F. Trunin et al., Acad. Sciences USSR, Izvestiya, Phys. Solid Earth, No. 1, 8 (1971); R. F. Trunin, Phys. Usp. 37, 1123 (1994).

[19] R. F. Trunin, Experimental Data on Shock Compression and Adiabatic Expansion of Condensed Matter (Sarov: RFNC-VNIIEF, 2001).

[20] R. F. Trunin, Shock Compression of Condensed Materials, (Camb. Univ. Press, 1998).

[21] The SESAME table for Aluminum compared in this case was \# 3718, given in S. P. Lyon and J. D. Johnson, Los Alamos National Laboratory Rep. LA-UR-92-3407, Los Alamos, NM (1992). For our analysis, differences between 
the various SESAME tables for aluminum are negligible.

[22] See for example J. A. Akins and T. J. Ahrens, Geophys.

Res. Lett. 29, 31 (2002) and references therein.
[23] M. Knaup et al., J. Phys. A 36, 6165 (2003) 